\title{
PARTICLE SIZE DETERMINATION IN IMPEDANCE FLOW CYTOMETRY USING MEASURED OPACITY
}

Douwe S. de Bruijn ${ }^{1}$, Koen F.A. Jorissen ${ }^{1}$, Wouter Olthuis ${ }^{1}$ and Albert van den Berg ${ }^{1}$

${ }^{1}$ BIOS Lab-on-a-Chip group, MESA+ institute, Max Planck Center for Complex Fluid Dynamics, University of Twente, P.O. Box 217, 7500 AE, Enschede, NETHERLANDS

\begin{abstract}
Microfluidic electrical flow cytometry is a popular method to study a wide variety of biological cell properties. Unfortunately, when using coplanar electrodes, this method is sensitive to positional differences of passing particles or cells.

In this work we present a novel compensation method to account for the particle position in a coplanar electrode setup using the measured electrical opacity.

We demonstrate an accurate size discrimination of 5, 6 and $7 \mu \mathrm{m}$ polystyrene beads irrespective of their position using the measured electrical opacity making use of the variation of electrical field strength with height in the channel. Thus, only two electrodes are required, which is favorable for microfluidic devices with size limitations.
\end{abstract}

\section{KEYWORDS}

Flow cytometry, size determination, electrical opacity, microfluidics, coplanar electrodes.

\section{INTRODUCTION}

Microfluidic electrical flow cytometry is a popular method to study label-free and non-invasively a wide variety of biological cell properties with high-throughput [1], [2]. Unfortunately, the particle position in these microfluidic electrical flow cytometers is a critical parameter for an accurate analysis of particles or cells [3]. Positional differences in coplanar electrode setups will significantly affect the impedance measurements, due to the nonhomogeneous electric field, as illustrated in Figure 1. This problem can be overcome by using plane-parallel electrodes or particle focusing, but this makes the chip fabrication more complex and expensive.

Previous studies have successfully compensated positional dependencies in nonhomogeneous electric fields using different metrics like transit times and prominence [4]-[6]. So far, these systems use at least three electrodes.

We propose a novel compensation method using the electrical opacity to account for the particle position using only two coplanar electrodes. Electrical opacity is defined as the impedance change at high over low frequency [7] and was recently correlated to the particle height in a microfluidic channel [8]. The closer the particle to the electrode, the larger the measured opacity, due to interactions with the capacitive double layer [8].

In this research, we deploy the measured opacity to correct for the particle position, resulting in an accurate estimate of the particle size using only two coplanar electrodes.

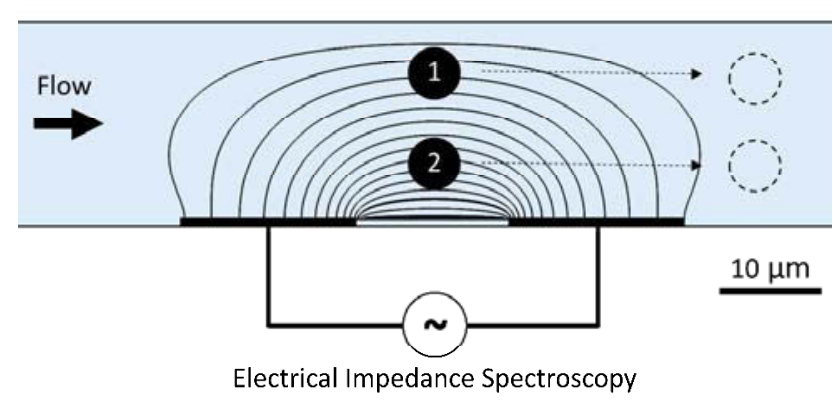

Figure 1: Representation of particles passing coplanar electrodes in a microfluidic flow cytometer at different heights. Two particles of equal size will induce a different change in impedance, due to different positions in the nonhomogenous electric field.

\section{THEORY}

In this section we explain how we use the measured opacity as a compensation method. For more details on the measured opacity and its relation to the particle position we refer to de Bruijn et al. [8].

The measured opacity $O$ is expressed as the change in impedance at high frequency over the change in impedance at low frequency [7]:

$$
O=\frac{\Delta|Z|_{\text {high freq }}}{\Delta|Z|_{\text {low freq }}}
$$

Furthermore, the electrical diameter $D(\mu \mathrm{m})$ of a passing spherical particle can be expressed as [5], [6]:

$$
D=G \Delta|Z|^{\frac{1}{3}}
$$

With $\mathrm{G}\left(\mu \mathrm{m} / \Omega^{1 / 3}\right)$ the gain factor of e.g. the circuitry and channel geometry and $\Delta|Z|(\Omega)$ the absolute impedance change. A linear relation between the measured opacity and the normalized measured electrical diameter was assumed:

$$
O=c_{1} n D+c_{2}
$$

With $c_{1}$ and $c_{2}$ fitting parameters and $n D$ the electrical diameter normalized for the nominal bead size. Conclusively, a correction of the measured particle diameter can be obtained using:

$$
D_{\text {corr }}=\frac{c_{1} D}{O-c_{2}}
$$

\section{METHOD \\ Device fabrication}

The measurements were performed using a simple coplanar electrode setup shown in Figure 2. The microfluidic chip consists of tantalum/platinum 
microelectrodes on glass and a PDMS layer with channels on top, as described previously [8], [9].

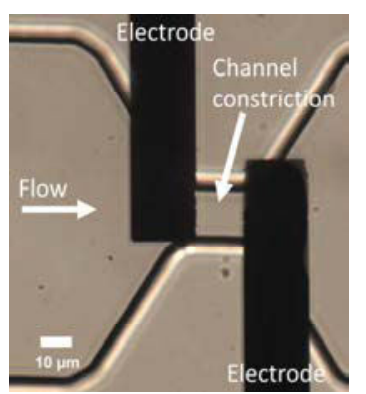

Figure 2: Top view of the microfluidic chip with two coplanar electrodes. The constriction channel reduces the volume between the electrodes and thereby increases the sensitivity. The fluidic channel is $20 \mu \mathrm{m}$ in height.

\section{Sample preparation}

Previous research with the same device was done on the study of algae and required seawater as medium [8]. The same protocols were used, therefore polystyrene beads were diluted in seawater (conductivity $=4 \mathrm{~S} / \mathrm{m}$ ) to a concentration of approximately $5 \cdot 10^{6}$ beads $/ \mathrm{ml}$. Beads of 5 , 6 and $7 \mu \mathrm{m}$ were used (Polysciences and Sigma Aldrich). Tween 20 was added to a concentration of $0.1 \%$ to prevent agglomeration and before each experiment 5 minutes of sonification was performed. Polystyrene beads have a density of $1.05 \mathrm{~g} / \mathrm{cm}^{3}$, which is similar to the density of seawater $\left(1.03 \mathrm{~g} / \mathrm{cm}^{3}\right)$, resulting in a uniform bead distribution throughout the channel.

The sample solution was introduced at a constant flow rate of $0.05 \mu \mathrm{l} / \mathrm{min}$ using a syringe pump (neMESYS, Cetoni).

\section{Data acquisition \& processing}

A lock-in amplifier (HF2LI, Zurich Instruments) with pre-amplifier (HF2TA, Zurich Instruments) was used to acquire impedance data at multiple frequencies (0.5 and 20 $\mathrm{MHz}$ ). The signal amplitude was set to $1 \mathrm{~V}$ peak to peak with a sampling rate of $28.8 \mathrm{kSa} / \mathrm{s}$.

After acquisition the data was post processed in Matlab R2020a (MathWorks). First, the baseline was removed using a polynomial fit, where after passing beads were registered using Matlab's 'findpeaks' function. A distinction was made between single peaks and $\mathrm{M}$-shaped peaks, as discussed in the results and Figure 3. The impedance change of M-shaped peaks was defined by the local minimum (II) between two peaks (I and III).

Fitting parameters $c_{1}$ and $c_{2}$ in eq. 3 were calculated using a robust fit. The accuracy of the compensation method was tested by comparing the standard deviation of the particle distributions with the manufacturer's specifications. The standard deviations were obtained using a Gaussian fit in Origin 2019b (OriginLab).

\section{RESULTS \& DISCUSSION}

A typical impedance response of two passing beads at different heights in the channel is shown in Figure 3. The first bead passes high in the channel and shows a single peak. The second bead shows an M-shaped response, due to a passage close to the electrodes [10]. The induced impedance change in region II (see inset Figure 3) was used for a fair comparison between passing beads at different heights. The measured opacity of the first bead was 0.20 , whereas the measured opacity of the second bead was 0.27 . This is as expected from literature: the closer to the electrodes, the larger the measured opacity, due to a shift in absolute impedance and frequency [8]. The reason for this is the interaction with the constant capacitive electrical double layer.

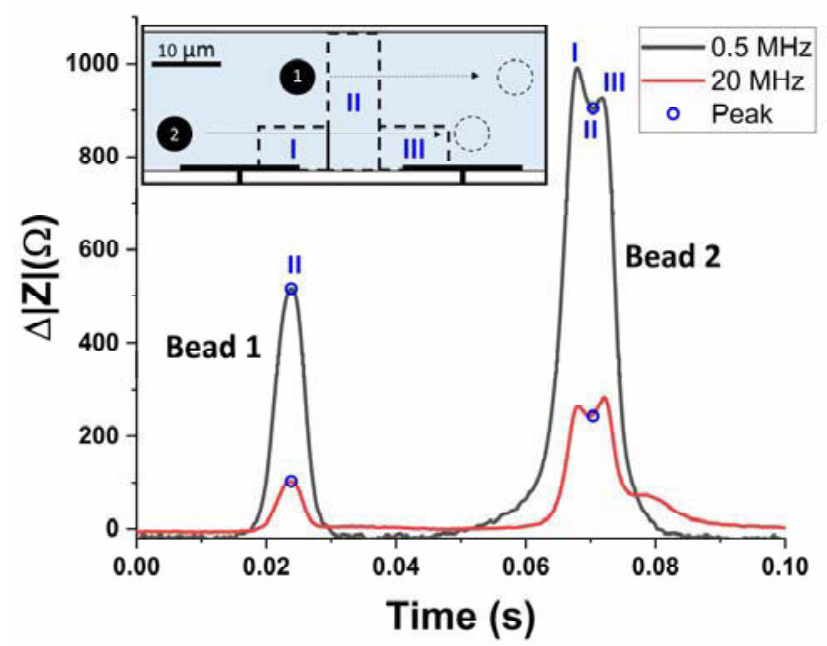

Figure 3: Impedance response at 0.5 and $20 \mathrm{MHz}$ of two 6 $\mu \mathrm{m}$ beads passing at different heights. The first bead passes higher in the channel than the second bead, resulting in a lower peak. The M-shaped response of the second bead can be attributed to sensitive areas, due to field-line inhomogeneity close to the electrodes as indicated in the inset [10]. The measured opacity of the first bead was 0.20 and of the second bead 0.27 .

The change in impedance at $20 \mathrm{MHz}$ versus $0.5 \mathrm{MHz}$ is displayed in Figure 4, showing a distinct separation between the 5, 6 and $7 \mu \mathrm{m}$ beads. On the contrary, we cannot separate the different bead sizes based on measurements at a single frequency, as illustrated in Figure 5 . In other words, we need the high frequency component to be able to differentiate.

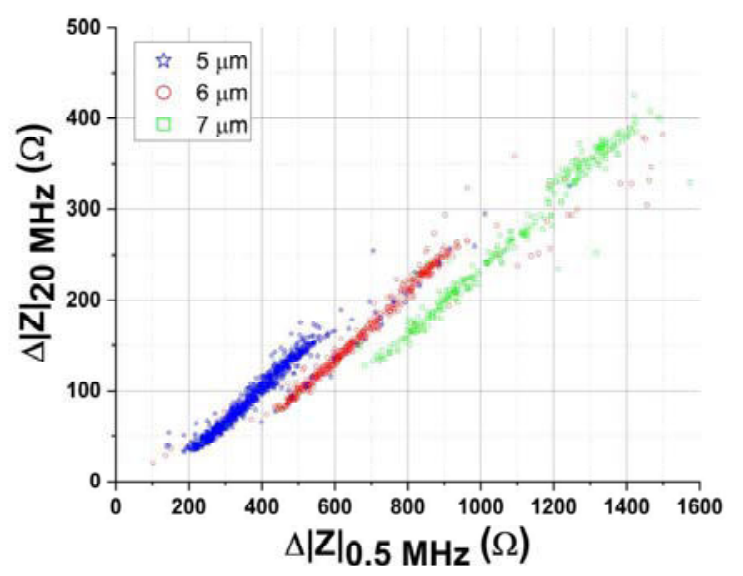

Figure 4: Impedance change at $20 \mathrm{MHz}$ versus $0.5 \mathrm{MHz}$ for three different experiments with different sized beads. Three distinct clusters can clearly be identified. 


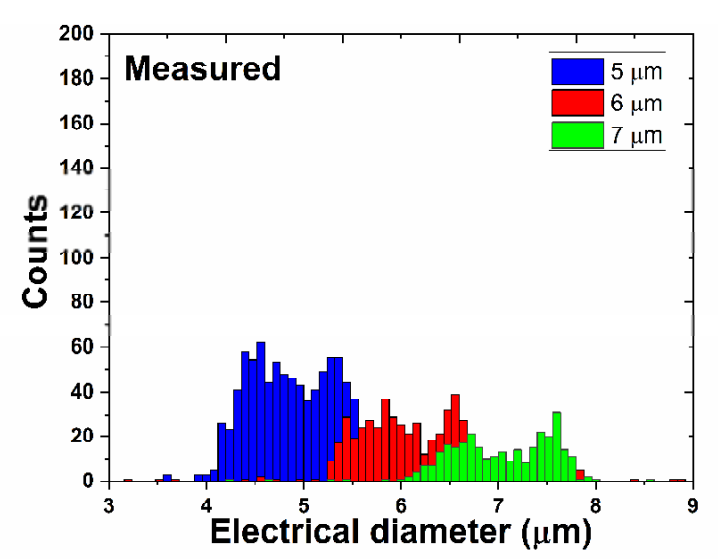

Figure 5: Electrical diameter (using 0.5 MHz signal) of the separately measured beads in Figure 4. The beads cannot fully be separated based on measurements at simply one frequency.

Next, we will improve the size distribution in Figure 5 using the measured opacity. The fitting parameters in eq. 3 were acquired using a robust fit, as visualized in Figure 6 and listed in Table 1.

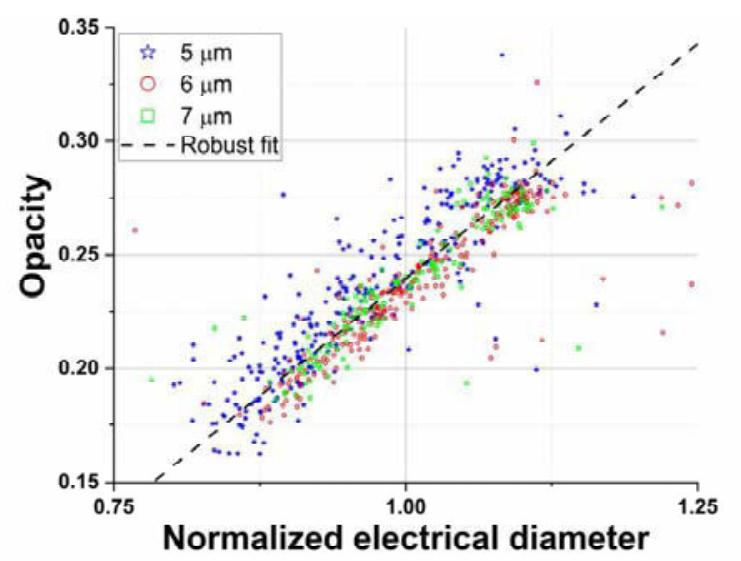

Figure 6: Measured opacity versus the electrical diameter normalized for the nominal bead size. The mean robust fit (eq. 3) is shown.

Table 1: Fitting parameters of eq. 3, calculated for all three bead sizes using a robust fit. The mean values are also shown in 'mean'.

\begin{tabular}{|l|c|c|}
\hline Diameter d $(\boldsymbol{\mu m})$ & $\mathbf{c}_{1}$ & $\mathbf{c}_{2}$ \\
\hline 5 & 0.40 & -0.15 \\
\hline 6 & 0.40 & -0.17 \\
\hline 7 & 0.39 & -0.15 \\
\hline Mean & $\mathbf{0 . 4 0}$ & $\mathbf{- 0 . 1 6}$ \\
\hline
\end{tabular}

Finally, the measured electrical diameter can be corrected using eq. 4 and the calculated mean fitting parameters. The result of the correction is shown in Figure 7.

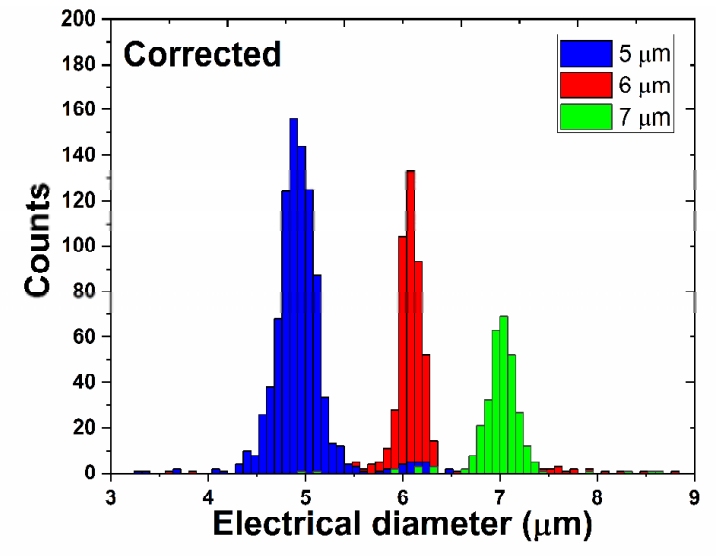

Figure 7: Measurements in Figure 5 corrected for the position in the microfluidic channel using the measured opacity (see eq. 4).

A mixture of 5, 6 and $7 \mu \mathrm{m}$ beads was introduced in our setup to test the accuracy of the proposed compensation method. The distribution of the measured and corrected bead diameters are shown in Figure 8. A Gaussian fit with three peaks at 5, 6 and $7 \mu \mathrm{m}$ resulted in standard deviations of $0.29 \mu \mathrm{m}(\mathrm{CV}=5.8 \%), 0.24 \mu \mathrm{m}(\mathrm{CV}=4.0 \%)$ and 0.20 $\mu \mathrm{m}(\mathrm{CV}=2.9 \%)$ respectively, which is slightly larger than the specifications of the manufacturers $(<2 \%,<10 \%$ and $<$ $2 \%)$.

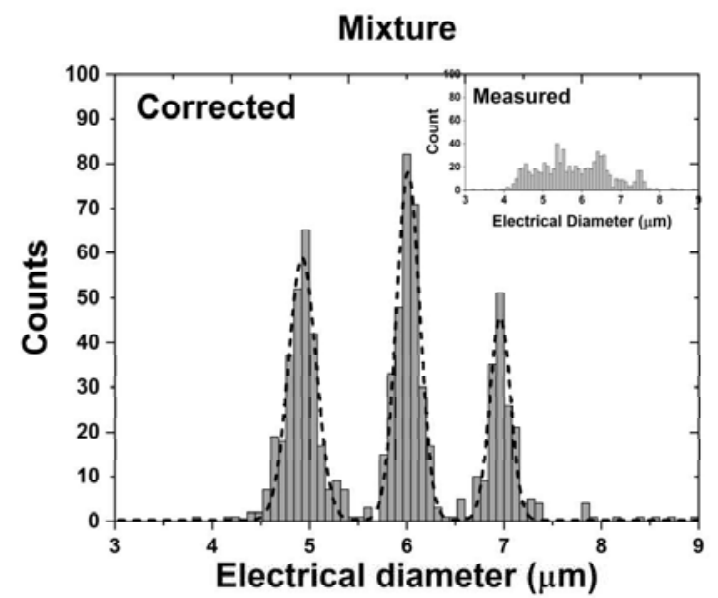

Figure 8: Results of the measured and corrected electrical diameter of a mixture of 5, 6 and $7 \mu \mathrm{m}$ beads. The Gaussian fit (dashed line) resulted in standard deviations of $0.29 \mu \mathrm{m}$ $(C V=5.8 \%), 0.24 \mu \mathrm{m}(\mathrm{CV}=4.0 \%)$ and $0.20 \mu \mathrm{m}(\mathrm{CV}=$ $2.9 \%$, respectively.

\section{CONCLUSION}

We have been able to determine the particle size of 5 , 6 and $7 \mu \mathrm{m}$ beads accurately in a nonhomogeneous electric field using only two coplanar electrodes and two frequencies. Therefore, the measured opacity can be deployed as a compensation method in simple coplanar electrode setups, which is a promising result for applications with size limitations. 


\section{ACKNOWLEDGEMENTS}

This work is part of the research programme of the Foundation for Fundamental Research on Matter (FOM), which is part of the Dutch Research Council (NWO). We would like to thank the Max Planck - Center for Complex Fluid Dynamics for support of this project.

\section{REFERENCES}

[1] C. Honrado, P. Bisegna, N. Swami, and F. Caselli, "Single-cell microfluidic impedance cytometry: from raw signals to cell phenotypes using data analytics," Lab Chip, vol. 21, no. 1, 2020.

[2] C. Petchakup, H. Li, and H. W. Hou, "Advances in single cell impedance cytometry for biomedical applications," Micromachines, vol. 8, no. 3, 2017.

[3] H. Daguerre, M. Solsona, J. Cottet, M. Gauthier, P. Renaud, and A. Bolopion, "Positional dependence of particles and cells in microfluidic electrical impedance flow cytometry: Origin, challenges and opportunities," Lab Chip, vol. 20, no. 20, pp. 3665-3689, 2020.

[4] D. Spencer, F. Caselli, P. Bisegna, and H. Morgan, "High accuracy particle analysis using sheathless microfluidic impedance cytometry," Lab Chip, vol. 16, no. 13, pp. 2467-2473, 2016.

[5] A. De Ninno, V. Errico, F. R. Bertani, L. Businaro, P. Bisegna, and F. Caselli, "Coplanar electrode microfluidic chip enabling accurate sheathless impedance cytometry," Lab Chip, vol. 17, no. 6, pp. 1158-1166, 2017.
[6] V. Errico, A. De Ninno, F. R. Bertani, L. Businaro, P. Bisegna, and F. Caselli, "Mitigating positional dependence in coplanar electrode Coulter-type microfluidic devices," Sensors Actuators, B Chem., vol. 247, pp. 580-586, 2017.

[7] R. A. Hoffman, T. S. Johnson, and W. B. Britt, "Flow cytometric electronic direct current volume and radiofrequency impedance measurements of single cells and particles," Cytometry, vol. 1, no. 6, pp. 377-384, 1981.

[8] D. S. de Bruijn, P. M. ter Braak, D. B. Van de Waal, W. Olthuis, and A. van den Berg, "Coccolithophore calcification studied by singlecell impedance cytometry: Towards single-cell PIC:POC measurements," Biosens. Bioelectron., p. $112808,2020$.

[9] B. de Wagenaar et al., "Spermometer: electrical characterization of single boar sperm motility," Fertil. Steril., vol. 106, no. 3, pp. 773-780.e6, 2016.

[10] J. Cottet, A. Kehren, H. van Lintel, F. Buret, M. Frénéa-Robin, and P. Renaud, "How to improve the sensitivity of coplanar electrodes and micro channel design in electrical impedance flow cytometry: a study," Microfluid. Nanofluidics, vol. 23, no. 1, pp. 1-11, 2019.

\section{CONTACT}

*Douwe de Bruijn, tel: +31534891935; 